\title{
TỐI ƯU HÓA BIÊN DẠNG DAO MÁY BĂM NHỰA DÙNG PHƯƠNG PHÁP TAGUCHI
}

\author{
NGUYỄN KHOA TRIỀU ${ }^{1}$, CHÂU MINH QUANG ${ }^{1}$, PHẠM ANH ĐỨC ${ }^{2}$, NGUYỄN NHẬT CƯỜNG ${ }^{1}$, \\ NGUYẼ̃N MINH PHĂNG ${ }^{1}$, MẠC VĂN ĐẠI, NGUYẼ̃N VĂN NINH \\ ${ }^{1}$ Khoa Co Khi Công Nghệ, Truờng Đại học Công nghiệp Thành phố Hồ Chí Minh, \\ ${ }^{2}$ Khoa Co Khí, Trường Đại học Đà Nẵng, \\ nguyenkhoatrieu@iuh.edu.vn
}

Tóm tắt. Bài báo này trình bày quá trình tối ưu hóa các thông số hình học của dao băm chai nhựa sử dụng mảng trực giao và phân tích phương sai (ANOVA). Đầu tiên, độ mòn hay độ biến dạng của dao được lựa chọn để đánh giá kết quả tối ưu hóa. Bảy thông số hình học chính của dao được lựa chọn, mỗi thông số có ba mức; riêng thông số vát mép chỉ có hai mức (có và không). Do đó, mảng trực giao $\mathrm{L}_{18}$ là phù hợp cho quy hoạch thực nghiệm trong nghiên cứu này. Phần mềm COMSOL $®$ được dùng để mô phỏng quá trình biến dạng của dao dựa trên mảng $\mathrm{L}_{18}$ này. Vì nhân tố vát mép gây nhiễu quá lớn tới kết quả mô phỏng, chỉ có dao được vát mép mới tiếp tục được dùng cho mô phỏng. Kết quả là chỉ còn lại sáu nhân tố đầu vào. Sau khi có kết quả từ 18 mô phỏng, ANOVA và tỷ số $\mathrm{S} / \mathrm{N}$ được dùng để tìm ra các mức thông số tối ưu và tác động của chúng tới đặc tính chất lượng đang khảo sát. Một mô phỏng bổ sung được thực hiện để chứng minh tính hiệu quả của phương pháp Taguchi. Kết quả thu được là $0.16209 \mu \mathrm{m}$, tối ưu hơn 18 kết quả đã thực hiện trước đó. Sau đó, một bộ dao với các thông số tối ưu được chế tạo và băm thử nghiệm. Kết quả độ mòn của các bộ dao trước và sau khi tối ưu hóa được đo bởi kính hiển vi điện tử ICamScope ${ }^{\circledR}$. Kết quả đo này xác nhận, dao tối ưu có độ mòn bé hơn. Từ đó, ta có thể kết luận rằng phương pháp Taguchi là một công cụ đơn giản nhưng hiệu quả cho tối ưu hóa quá trình cho một công nghệ truyền thống như băm / nghiền chai nhựa. Kết cấu dao tìm được cứng chắc, không bị biến dạng dẻo như dao băm đã công bố, ảnh hưởng xấu tới quá trình băm.

Từ khóa. Máy băm nhựa, Dao băm, Tối ưu hóa, Taguchi, ANOVA.

\section{A TAGUCHI NUMERICAL AND EXPERIMENTAL OPTIMIZATION OF GEOMETRICAL PARAMETERS OF SHREDDER BLADE FOR PLASTIC BOTTLE SHREDDER}

\begin{abstract}
The objective of this investigation is to optimize geometrical parameters of shredder blade for plastic bottle shredder utilizing Taguchi orthogonal array and analysis of variance (ANOVA). As the first step, the wear or deformation of the plastic shredder was selected as the quality of characteristic to be optimized. Seven geometrical parameters of the blade were then chosen to be analyzed as the influencing factors toward the wear for optimization purpose. Three levels of each factor (except chamfer factor) were considered for a better understanding of the non-linear effects of them. Hence $\mathrm{L}_{18}$ orthogonal array was suitable for interpretation of all seven factors. As the next step, a numerical investigation of the deformation of the blade was carried out by utilizing this array via COMSOL®. Because the chamfer factor caused too much noise to the wear results, only chamfered blades were considered in this study. Therefore the orthogonal array was reconstructed with six remaining factors. As the next step, 18 numerical simulations were carried out by utilizing the combination of process parameters based on this $\mathrm{L}_{18}$ array. Then, the S/N ratio and ANOVA were used to find the optimal levels to indicate the impact of the geometry parameters on the deformation results. A verification simulation was also performed to demonstrate the effectiveness of the Taguchi method, resulting in a lower deformation $(0.16209 \mu \mathrm{m})$ than that of any previous 18 results. A new set of the optimized blade was fabricated and experimented. An ICamScope ${ }^{\circledR}$ microscope was used to measure the wear of both before and after optimized blades, showing slightly lower wear. Hence, it can be concluded that Taguchi method is a simple but effective tool to optimize such a traditional technology as plastic bottle shredding. The improved blade was found to be firm and free of plastic deformation.
\end{abstract}

Keywords. Plastic shredder, Shredder blade, Optimization, Taguchi, ANOVA. 


\section{GIỚI THIỆ}

Ngày nay, nhựa đã trở thành một trong những vật liệu được sử dụng phổ biến nhất trên thế giới [1]. Do đó, chất thải nhựa đã trở thành mối đe dọa lớn đối với môi trường do thời gian phân hủy dài, lượng chất thải nhựa khổng lồ, không phân hủy sinh học và làm cạn kiệt tài nguyên thiên nhiên [2]. Do đó, nhu cầu tái chế nhựa, trong đó một loại số nhựa được xử lý để tái sử dụng, đang tăng lên từng ngày. Tái chế là một trong những hành động cần thiết hứa hẹn nhất để giảm tác động môi trường của chất thải nhựa, vì nó làm giảm lượng khí thải $\mathrm{CO}_{2}$, sử dụng dầu và lượng chất thải cần được xử lý [3]. Khi tái chế nhựa thải, để tăng cường tính di động, có thể dễ dàng và sẵn sàng sử dụng trong các sản phẩm mới khác, nhựa thải phải được giảm kích thước thành các hạt nhỏ hơn bằng máy băm nhựa.

Do tầm quan trọng của quá trình băm nhỏ, đã có nhiều nghiên cứu liên quan đến thiết kế và phát triển máy băm nhựa. Ayo, Olukunle, và Adelabu từ Nigeria [2] đã phát triển một máy băm nhựa thải giá rẻ cho các nhà máy quy mô vừa và nhỏ chuyên xử lý nhựa tái chế. Máy cũng được đánh giá ở ba tốc độ làm việc về kích thước hạt trung bình. Reddy và Raju [4] cũng chế tạo một máy băm nhựa mini. Họ viết rằng máy rất đơn giản, hiệu quả và chi phí thấp. Tuy nhiên, lưỡi dao và điều kiện làm việc của máy chưa được phân tích. Cũng như vậy, các công trình nghiên cứu trong [5-11] có cùng tình huống là các máy chưa được đánh giá về hiệu suất. Cùng lúc đó, Jassi và cộng sự [12] thiết kế và chế tạo máy băm/máy nghiền và máy đùn cho hệ thống tái chế nhựa của họ. Farayibi và cộng sự. [3] cũng trình bày một thiết kế cho một máy tái chế nhựa. Sau đó, phân tích cấu trúc được thực hiện bằng công cụ phân tích phần tử hữu hạn (FEA) trong ứng dụng SolidWorks. Tuy nhiên, ứng suất cực đại thu được đóng góp không nhiều cho sự phát triển và đánh giá máy.

Trong máy băm nhựa, lưỡi dao băm đóng vai trò quan trọng trong quá trình băm nhỏ vì nó quyết định kích thước của các mảnh vụn. Hơn nữa, nó là một yếu tố quan trọng ảnh hưởng đến hiệu quả làm việc và tuổi thọ của máy [13]. Tuy nhiên, các thông số hình học của dao cắt và ảnh hưởng của chúng đối với sự mài mòn và/hoặc biến dạng chưa được nghiên cứu đủ. Trong [13], các tác giả đã tiến hành phân tích biến dạng và phân tích ứng suất tương đương. Tuy nhiên, những kết quả này đã không được sử dụng để đánh giá hoặc tối ưu hóa thêm. Theo cách tương tự, Sekar [14] đã thực hiện phân tích tổng biến dạng, mối tương quan giữa ứng suất và biến dạng bằng phương pháp cấu trúc tĩnh. Sau đó, lưỡi cắt được cải tiến bằng phương pháp làm cứng hoặc mạ crôm hoặc tăng các thuộc tính của lưỡi cắt. Nasr và Yehia từ $\mathrm{Ai}$ Cập [15] và Yepes cùng các cộng sự từ Columbia [16] cũng đã phân tích ứng suất tĩnh trong một lưỡi cắt cho trước với ba và hai lưỡi cắt tương ứng, được sử dụng để băm nhỏ nhựa thải PET (polyetylen terephthalate). Và trong [17], các tác giả chỉ cần kiểm tra các biến thể của phân phối ứng suất ở các giá trị lực khác nhau bằng SolidWorks ${ }^{\circledR}$ mà không sử dụng thêm thông tin thu được này.

Do thiếu sót này, một quy trình tối ưu hóa dùng phương pháp Taguchi đã được áp dụng trong nghiên cứu này. Phương pháp Taguchi là kỹ thuật thường được sử dụng để thu được các giá trị tối ưu của các tham số liên quan [18]. Phương pháp Taguchi trước tiên được áp dụng với việc xem xét các tham số hình học quan trọng của lưỡi cắt, dẫn đến các giá trị tối ưu. Từ đó, các tác động của các tham số hình học đối với sự hao mòn/biến dạng của lưỡi cắt được nghiên cứu bằng cách sử dụng tổ hợp mà đem đến kết quả hao mòn/biến dạng tối thiểu.

\section{PHƯƠNG PHÁP VÀ VẠT LIỆ}

\subsection{Mô tả lưỡi cắt}

Trong nghiên cứu này, hiện tượng biến dạng/mài mòn của lưỡi cắt của máy băm rác thải nhựa như minh họa trong Hình 1, đã được nghiên cứu. Máy băm nhựa, là loại trục đơn, bao gồm 14 lưỡi cắt như trong Hình 2. Hình $2 \mathrm{a}$ cho thấy thiết kế của lưỡi cắt. Nó được thiết kế để có hình chữ $\mathrm{S}$ với hai đầu băm và một lỗ hình lục giác ở trung tâm để cố định với trục. Hình $2 \mathrm{~b}$ cho thấy một bức ảnh của lưỡi dao băm được chế tạo dùng phương pháp cắt laser và mài.

Vật liệu được sử dụng cho lưỡi cắt là thép $\mathrm{S} 45 \mathrm{C}$ có độ dày $5 \mathrm{~mm}$. Vật liệu được chọn vì lý do giảm chi phí, phù hợp với các xưởng sản xuất quy mô nhỏ. Trước khi gia công tinh, các lưỡi dao đã được xử lý nhiệt để có các đặc tính tốt hơn. Phương pháp tôi thể tích đã được sử dụng cho các lưỡi dao trong nghiên cứu này bởi một công ty địa phương. Bảng 1 cho thấy các tính chất quan trọng của hai giai đoạn này của vật liệu được sử dụng trong mô phỏng. 


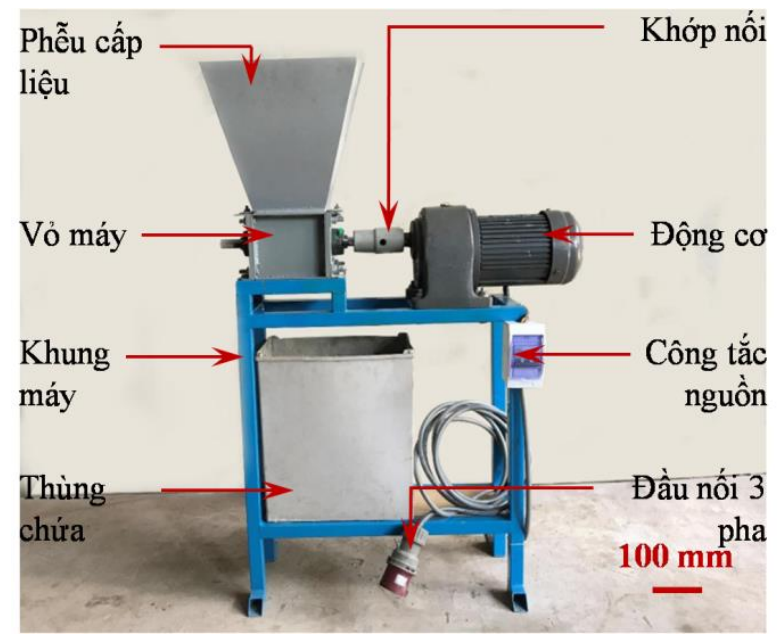

Hình 1. Hình ảnh của máy băm chai nhựa được phát triển trong nghiên cứu này
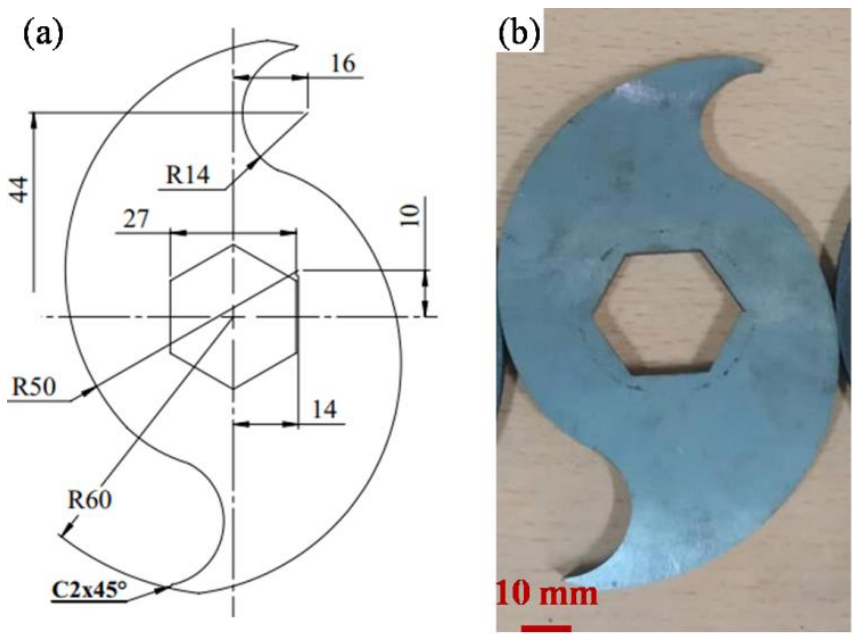

Hình 2 (a) minh họa về lưỡi dao băm (b) hình ảnh của lưỡi dao băm được chế tạo

Bảng 1: Tính chất vật liệu của thép $\mathrm{S} 45 \mathrm{C}$ trước và sau khi xử lý nhiệt được sử dụng trong mô phỏng.

\begin{tabular}{clccc}
\hline TT & \multicolumn{1}{c}{ Tính chất } & Trước khi xử lý nhiệt & Sau khi xử lý nhiệt & Đơn vị \\
\hline 1 & Khối lượng riêng & $7.78 \times 10^{3}$ & $7.78 \times 10^{3}$ & $\mathrm{~kg} / \mathrm{m}^{3}$ \\
2 & Young's modulus (E) & 205 & 460 & $\mathrm{GPa}$ \\
3 & Hệ số Poisson & 0.3 & 0.3 & - \\
4 & Hệ số giãn nở nhiệt & 12.5 & 12.5 & $1 / \mathrm{K}$ \\
\hline
\end{tabular}

Cho mô phỏng, một mô hình hình học $2 \mathrm{D}$ đã được xây dựng cho toàn bộ lưỡi dao băm. Trong nghiên cứu này, các phần tử tam giác đã được sử dụng để phân chia hữu hạn miền tính toán. Mô hình được xây dựng với 9892 phần tử tam giác, 376 phần tử cạnh và 20 phần tử đỉnh.

Cần lưu ý rằng trong khi lưỡi dao băm này cũng có hình chữ $\mathrm{S}$ giống như loại lưỡi băm đã công bố, phần đầu của nó đã được cải tiến. Đầu của lưỡi dao được tạo bởi hai đường cong, sau đó được cắt theo đường chéo, như thể hiện trong phần chi tiết trong Hình 4, để tăng độ cứng của đẩu lưỡi. Đồng thời, đường cắt chéo này cho phép mài lưỡi băm mà không cần tháo lưỡi băm ra, rút ngắn thời gian bảo dưỡng, sửa chữa. Hơn nữa, quá trình mài sắc không làm thay đổi đường kính ngoài của lưỡi dao, kích thước R60 trong Hình 2a. Ở các loại lưỡi dao chưa cải tiến, chai nhựa có khả năng mắc vào khe hở giữa lưỡi dao và thành máy hủy khi đường kính ngoài của lưỡi dao bị thay đổi.

\subsection{Phương pháp Taguchi}

Ngày nay, phương pháp Taguchi ngày càng được ứng dụng rộng rãi trong lập quy hoạch thực nghiệm [19]. Phương pháp này góp phần cải thiện năng suất trong quá trình nghiên cứu và phát triển, tạo ra các sản phẩm có chất lượng cao mà giá thành thấp. Nhà nghiên cứu Taguchi đã phát triển phương pháp dựa trên thí nghiệm mảng trực giao (Orthogonal Array) nhằm giảm các tham số của thí nghiệm với sự bố trí thích hợp các điều kiện thuận lợi nhất của các tham số điều khiển được. Các mảng trực giao tạo ra một sự cân bằng giữa các thí nghiệm và tỉ số Signal - to - Noise $(\mathrm{S} / \mathrm{N})$ là hàm logarit của đầu ra mong muốn, thỏa mãn mục đích tối ưu hóa, giúp cho việc phân tích và dự đoán kết quả. Trong phương pháp Taguchi thuật ngữ "Signal" để chỉ giá trị trung bình (giá trị mong muốn) của mục tiêu đầu ra và thuật ngữ "Noise" để chỉ giá trị không mong muốn. Do vậy tỉ số $\mathrm{S} / \mathrm{N}$ được sử dụng để tính sự sai lệch so với giá trị mong muốn [20,21].

Tỉ số S/N để tối ưu hóa:

Nhỏ hơn-tốt hơn:

$$
\mathrm{S} / \mathrm{N}=-10 \mathrm{x} \log _{10}\left[(1 / \mathrm{n}) \mathrm{x} \sum\left(\mathrm{y}_{\mathrm{i}}^{2}\right)\right]
$$

Lớn hơn - tốt hơn:

$$
\mathrm{S} / \mathrm{N}(\mathrm{L})=-10 \mathrm{x} \log _{10}\left[(1 / \mathrm{n}) \mathrm{x} \sum\left(1 / \mathrm{y}_{\mathrm{i}}^{2}\right)\right]
$$


Thiết kế của các thí nghiệm sử dụng phương pháp Taguchi là hiệu quả hơn so với phương pháp thống kê khác. Bằng cách chọn đúng mức độ phù hợp của các biến độc lập khác nhau, số lượng các thí nghiệm được giảm đáng kể. Cùng lúc đó, không mất mát bất kỳ thông tin nào do giảm số thí nghiệm. Mục tiêu của Taguchi đã giảm thiểu sự thay đổi xung quanh mục tiêu và nâng cao chất lượng. Việc sử dụng và học tập kỹ thuật này cho phép các kỹ sư, nhà khoa học và nghiên cứu cần ít thời gian cho quy hoạch thực nghiệm và phân tích kết quả hơn.Với những cải tiến trong công nghệ máy tính, nó đang trở thành dễ dàng hơn để sử dụng phương pháp Taguchi trong các ứng dụng. Phổ biến nhất là phân tích kỹ thuật dùng các phần mềm máy tính [21].

\subsection{Lưu đồ quy trình tối ưu hóa dùng phương pháp Taguchi}

Lưu đồ quy trình tối ưu hóa được thực hiện như minh họa trong Hình 3. Quy trình cơ bản gồm hai bước là mô phỏng và kiểm nghiệm bằng thực nghiệm.

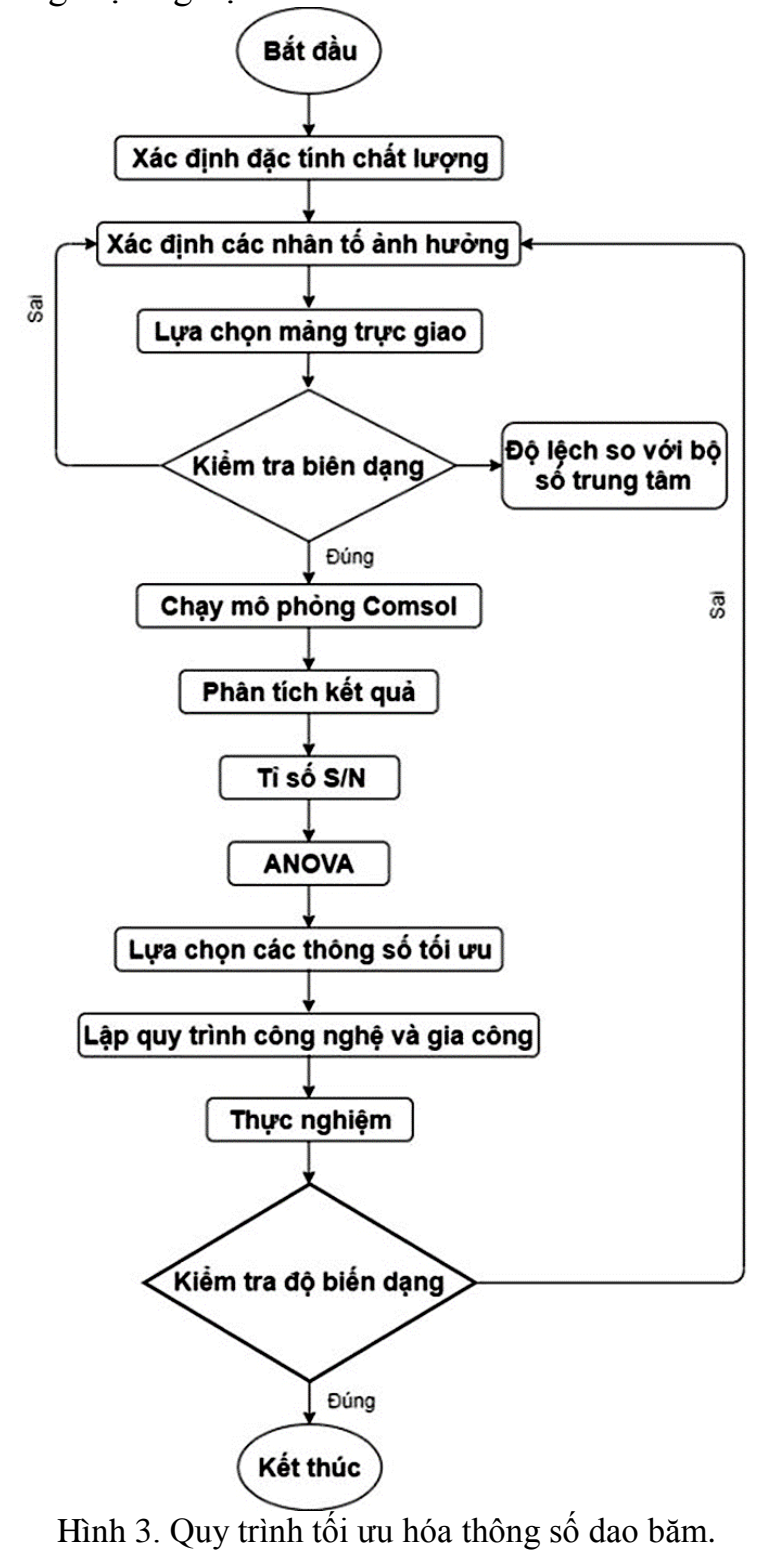

\section{MÔ PHỎNG}

Hình 3. Quy trình tối ưu hóa thông số dao băm.

Trong nghiên cứu hiện tại, một phần mềm phân tích vật lý, Comsol ${ }^{\circledR}$, đã được sử dụng để phân tích biến dạng của lưỡi dao. Trong quá trình băm nhỏ, sự biến dạng của lưỡi dao băm có nguồn gốc từ trở lực của nhựa vì các chai nhựa được cắt nhỏ thành từng mảnh nhỏ. Để phân tích chính xác hiện tượng biến dạng, phần mềm phân tích sử dụng mô hình rắn đàn hồi tuyến tính (loại đẳng hướng) với vật liệu đàn hồi tuyến tính. Các phương trình được trình bày dưới đây:

$-\nabla \cdot \sigma=F v$ 


$$
\sigma=s
$$

Định luật Duhamel-Hooke mô tả mối quan hệ giữa ten-xơ ứng suất với ten-xơ biến dạng và và nhiệt độ:

$$
s-s_{0}=C:\left(\varepsilon-\varepsilon_{0}-\alpha \theta\right)
$$

Ten-xơ của tổng biến dạng căng được mô tả theo građien của biến dạng:

$$
\varepsilon=\frac{1}{2}\left[(\nabla u)^{T}+\nabla u\right]
$$

trong đó $\mathrm{Fv}$ là lực trên một đơn vị thể tích, $\mathrm{s}_{0}$ và $\varepsilon_{0}$ là ứng suất và biến dạng ban đầu, $\mathrm{C}$ là tenxơ đàn hồi bậc 4, ":" là ten-xơ kép, $\alpha$ là ten-xơ giãn nở nhiệt, và $\theta=T-T_{\text {ref. }}$.

\section{KẾT QUẢ VÀ THẢO LUẬN}

\subsection{Lựa chọn các tham số quá trình và mảng trực giao}

Có nhiều kích thước tạo thành hình dạng của lưỡi dao băm. Tuy nhiên, chỉ các kích thước hình thành các đầu băm được xem xét trong cách tiếp cận ban đầu hướng tới tối ưu hóa. Cần lưu ý rằng đường kính ngoài của lưỡi dao, $120 \mathrm{~mm}$, được duy trì trong nghiên cứu này. Như một sự tiếp nối của các công trình trước đó, nghiên cứu hiện tại đã sử dụng lại máy băm nhựa, ngoại trừ các lưỡi dao băm. Hơn nữa, ba cấp độ của từng yếu tố được thiết lập dựa trên các thử nghiệm sơ bộ. Bởi vì các kích thước ràng buộc lẫn nhau, các mức trong Bảng 2 đã được chọn để lưỡi dao băm vẫn có hình chữ $\mathrm{S}$. Bảng 2 liệt kê các kích thước và ba cấp độ của chúng được sử dụng trong nghiên cứu này. Sử dụng phương pháp này, một nghiên cứu dựa trên DOE và mô hình bề mặt đáp ứng có thể được xây dựng một cách có hệ thống.

\begin{tabular}{cclcccc}
\multicolumn{7}{c}{ Bảng 2: Các thông số hình học của dao băm. } \\
\hline TT & Ký hiệu & Thông số & Mức 1 & Mức 2 & Mức 3 & Đơn vị \\
\hline 1 & A & Đường kính $\Phi 28$ & 27.8 & 28 & 28.2 & $\mathrm{~mm}$ \\
2 & B & Tọa độ x của $\Phi 28$ & 15.8 & 16 & 16.2 & $\mathrm{~mm}$ \\
3 & C & Tọa độ y của $\Phi 28$ & 43.8 & 44 & 44.2 & $\mathrm{~mm}$ \\
4 & D & Đường kính $\Phi 100$ & 99.8 & 100 & 100.2 & $\mathrm{~mm}$ \\
5 & E & Tọa độ x của $\Phi 100$ & 13.8 & 14 & 14.2 & $\mathrm{~mm}$ \\
6 & F & Tọa độ y của $\Phi 100$ & 9.8 & 10 & 10.2 & $\mathrm{~mm}$ \\
\hline
\end{tabular}

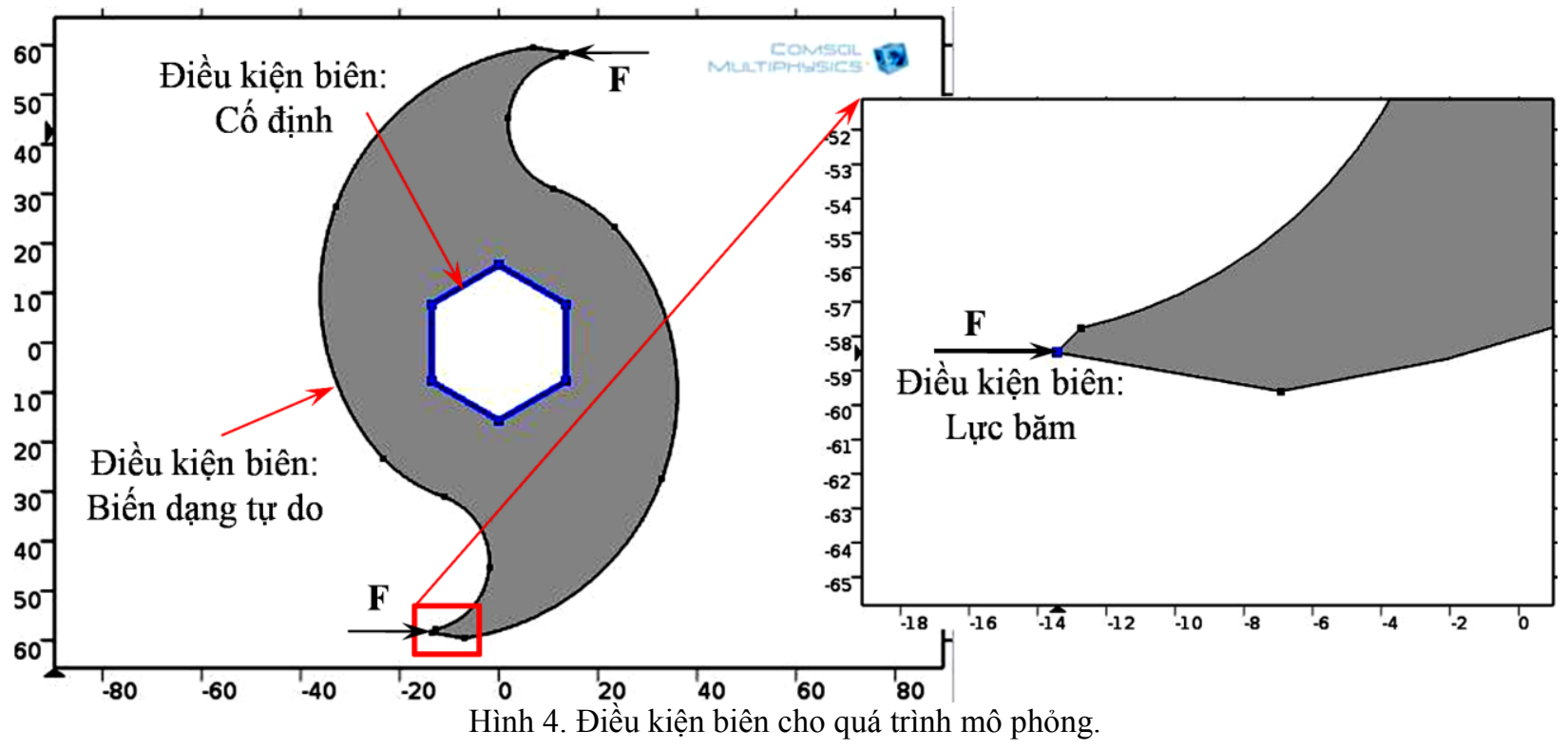

Từ số lượng kích thước đã chọn và cấp độ tương ứng của chúng, một tập hợp con của mảng trực giao $\mathrm{L}_{18}$ $\left(3^{6}\right)$ đã được chọn như trong Bảng 3. 18 mô phỏng số cho các lưỡi dao dưới tác động của lực băm được thực hiện bằng cách sử dụng bộ kích thước trong mảng trực giao. Các điều kiện biên chính của mô phỏng được trình bày trong Hình 4 . Trong đó, $\mathrm{F}$ là lực cắt tác động lên đầu mũi dao. Thông qua các tính toán lý thuyết từ độ bền của chai nhựa $\mathrm{PET}$, độ rộng của dao, đường kính của chai nhựa $\mathrm{PET}$, độ dày của chai, $\mathrm{F}$ có giá trị $1809.54 \mathrm{~N}$. Để đơn giản hóa quá trình mô phỏng, lực cắt được đặt theo phương tiếp tuyến với trục dao. Mô phỏng được thực hiện cho dao trong quá trình hoạt động liên tục, do đó, cả hai đầu mũi dao 
đều chịu tác động của lực $\mathrm{F}$, ngược chiều nhau. Điều kiện biên cho trục lục giác là cố định, không bị biến dạng. Tất cả các phần còn lại của dao đều tự do biến dạng.

Biến dạng tối đa của các lưỡi dao, được dự đoán bằng các mô phỏng số và tỷ lệ $\mathrm{S} / \mathrm{N}$ tương ứng cũng được liệt kê trong Bảng 3. Vì biến dạng, đặc tính mục tiêu của nghiên cứu, cần được giảm thiểu để đạt được quá trình băm nhỏ hiệu quả, công thức tỷ lệ $\mathrm{S} / \mathrm{N}$ "nhỏ là tốt" được sử dụng để xác định từng tỷ lệ $\mathrm{S} / \mathrm{N}$.

Bảng 3: Cấu trúc mảng trực giao $\mathrm{L}_{18}$.

\begin{tabular}{cccccccccc}
\hline STN & A & B & C & D & E & F & Độ biến dạng & MSD & S/N \\
\hline 1 & 1 & 1 & 1 & 1 & 1 & 1 & 0.1883 & 0.0355 & 14.5029 \\
2 & 1 & 2 & 2 & 2 & 2 & 2 & 0.1780 & 0.0317 & 14.9909 \\
3 & 1 & 3 & 3 & 3 & 3 & 3 & $\mathbf{0 . 1 6 5 3}$ & 0.0273 & 15.6364 \\
4 & 2 & 1 & 1 & 2 & 2 & 3 & 0.1774 & 0.0314 & 15.0239 \\
5 & 2 & 2 & 2 & 3 & 3 & 1 & 0.1851 & 0.0343 & 14.6522 \\
6 & 2 & 3 & 3 & 1 & 1 & 2 & 0.1741 & 0.0303 & 15.1818 \\
7 & 3 & 1 & 2 & 1 & 3 & 2 & 0.1917 & 0.0368 & 14.3471 \\
8 & 3 & 2 & 3 & 2 & 1 & 3 & 0.1721 & 0.0296 & 15.2859 \\
9 & 3 & 3 & 1 & 3 & 2 & 1 & 0.1792 & 0.0321 & 14.9347 \\
10 & 1 & 1 & 3 & 3 & 2 & 2 & 0.1820 & 0.0331 & 14.7979 \\
11 & 1 & 2 & 1 & 1 & 3 & 3 & 0.1749 & 0.0306 & 15.1440 \\
12 & 1 & 3 & 2 & 2 & 1 & 1 & 0.1702 & 0.0290 & 15.3787 \\
13 & 2 & 1 & 2 & 3 & 1 & 3 & 0.1754 & 0.0308 & 15.1186 \\
14 & 2 & 2 & 3 & 1 & 2 & 1 & 0.1957 & 0.0383 & 14.1660 \\
15 & 2 & 3 & 1 & 2 & 3 & 2 & 0.1773 & 0.0314 & 15.0263 \\
16 & 3 & 1 & 3 & 2 & 3 & 1 & 0.2019 & 0.0408 & 13.8986 \\
17 & 3 & 2 & 1 & 3 & 1 & 2 & 0.1772 & 0.0314 & 15.0283 \\
18 & 3 & 3 & 2 & 1 & 2 & 3 & 0.1760 & 0.0310 & 15.0914 \\
\hline
\end{tabular}

\subsection{Kết quả $\mathrm{S} / \mathrm{N}$}

Bảng 4 trình bày kết quả tỷ lệ $\mathrm{S} / \mathrm{N}$ được tóm tắt cho từng yếu tố. Các kết quả cũng được vẽ trong Hình 5 , minh họa rõ ràng ảnh hưởng của các yếu tố. Ví dụ, độ dịch chuyển của đầu băm của lưỡi dao băm tăng lên khi đường kính tăng $\Phi 28$ (yếu tố $\mathrm{A}$ ), tọa độ $\mathrm{x}$ của $\Phi 100$ (yếu tố $\mathrm{E}$ ) và giảm tọa độ $\mathrm{x}$ của $\Phi 28$ (yếu tố $\mathrm{B}$ ), tọa độ y của $\Phi 100$ (yếu tố $\mathrm{F}$ ) trong phạm vi sử dụng điển hình. Lý do là khi những yếu tố này thay đổi như trên, phần đầu của lưỡi dao trở nên nhỏ hơn và mỏng hơn. Kết quả là, ứng suất ở đầu lưỡi dao tăng lên. Do đó, sự biến dạng đã tăng lên tương ứng. Bằng cách xem xét sự khác biệt giữa các giá trị tỷ lệ $\mathrm{S} / \mathrm{N}$ tối đa và tối thiểu, sự đóng góp của từng tham số cũng đã tính được. Hình 6 cho thấy biểu đồ đóng góp của các yếu tố. Như được hiển thị, tọa độ $\mathrm{y}$ của $\Phi 100$ (yếu tố $\mathrm{F}$ ) là yếu tố quan trọng nhất ảnh hưởng đến độ biến dạng.

Bảng 4: Kết quả tỷ số $\mathrm{S} / \mathrm{N}$ cho mỗi thông số cài đặt.

\begin{tabular}{|c|c|c|c|c|c|c|c|c|}
\hline \multicolumn{2}{|l|}{ Yếu tố } & A & B & $\mathrm{C}$ & $\mathrm{D}$ & $\mathrm{E}$ & $\mathrm{F}$ & Tống cộng \\
\hline \multirow{3}{*}{$\begin{array}{l}\text { Tổng tỷ } \\
\text { lệ SN }\end{array}$} & Mức 1 & 15.0751 & 14.6149 & 14.9433 & 14.7389 & 15.082 & 14.5888 & \multirow{3}{*}{44.7009} \\
\hline & Mức 2 & 14.8615 & 14.8779 & 14.9298 & 14.9341 & 14.8341 & 14.8954 & \\
\hline & Mức 3 & 14.7643 & 15.2082 & 14.8278 & 15.0280 & 14.7841 & 15.2167 & \\
\hline \multicolumn{2}{|c|}{ Độ chênh lệch } & 0.3108 & 0.5934 & 0.1156 & 0.2892 & 0.2986 & 0.6279 & 2.2353 \\
\hline \multicolumn{2}{|c|}{ Phần trăm \% } & 13.90 & 26.54 & 5.17 & 12.94 & 13.36 & 28.09 & 100 \\
\hline
\end{tabular}




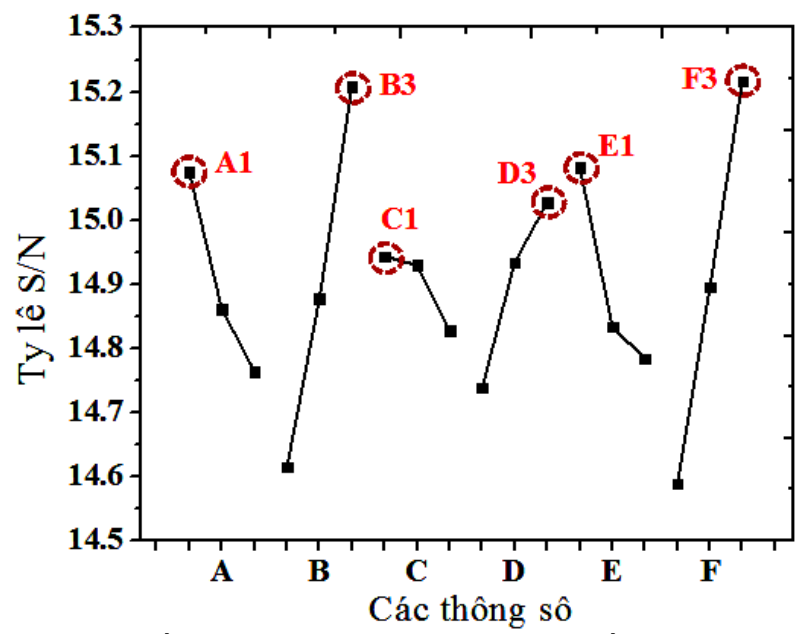

Hình 5. Kết quả phân tích tỷ lệ $\mathrm{S} / \mathrm{N}$ cho thấy ảnh hưởng của các tham số đến độ biến dạng

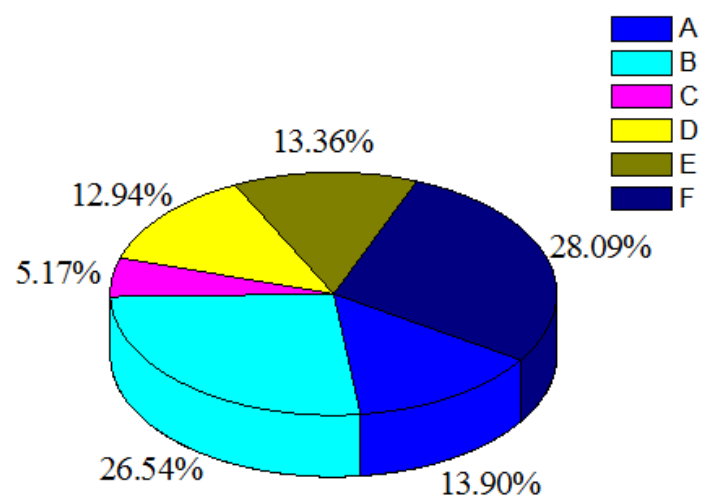

Hình 6. Biểu đồ mức đóng góp của từng tham số dựa trên phân tích tỷ lệ $\mathrm{S} / \mathrm{N}$

\subsection{Kết quả phân tích ANOVA}

Để nghiên cứu thêm sự đóng góp của các nhân tố liên quan, phương pháp ANOVA đã được thực hiện như trong Bảng 5. Phù hợp với phân tích tỷ lệ $\mathrm{S} / \mathrm{N}$, tọa độ $\mathrm{x}$ của $\Phi 28$ (nhân tố $\mathrm{B}$ ) và tọa độ $\mathrm{y}$ của $\Phi 100$ (nhân tố $\mathrm{F}$ ) là các nhân tố quan trọng liên quan đến việc giảm biến dạng. Có một sự khác biệt nhỏ về mức độ đóng góp của các nhân tố mặc dù có một xu hướng tương tự giữa tỷ số $\mathrm{S} / \mathrm{N}$ và các phân tích $\mathrm{ANOVA}$. Các phân tích ANOVA cho thấy bốn nhân tố, đường kính $\Phi 28$ (nhân tố $\mathrm{A}$ ), tọa độ $\mathrm{x}$ của $\Phi 100$ (nhân tố $\mathrm{E}$ ), tọa độ $\mathrm{x}$ của $\Phi 28$ (nhân tố $\mathrm{B}$ ) và tọa độ $\mathrm{y}$ của $\Phi 100$ (nhân tố $\mathrm{F}$ ) có giá trị $\mathrm{F}$ lớn hơn $\mathrm{F}(0,05,2,5)$, do đó biều thị rằng chỉ những yếu tố này có ý nghĩa thống kê.

\begin{tabular}{lcrrrrcc}
\multicolumn{8}{c}{ Bảng 5: Bảng kết quả ANOVA. } \\
\hline & S & \multicolumn{1}{c}{$\mathrm{f}$} & $\mathrm{V}$ & $\mathrm{F}$ & $\mathrm{F}_{(0.01,2,7)}$ & $\mathrm{P} \%$ & Xếp hạng \\
$\mathrm{A}$ & $1.3357 \mathrm{E}-04$ & 2 & $6.6785 \mathrm{E}-05$ & 6.1504 & 5.78614 & 9.14 & 4 \\
$\mathrm{~B}$ & $4.6493 \mathrm{E}-04$ & 2 & $2.3247 \mathrm{E}-04$ & 21.4082 & 5.78614 & $\mathbf{3 1 . 8 1}$ & 2 \\
$\mathrm{C}$ & $2.8001 \mathrm{E}-05$ & 2 & $1.4000 \mathrm{E}-05$ & 1.2893 & 5.78614 & 1.92 & \\
$\mathrm{D}$ & $1.1502 \mathrm{E}-04$ & 2 & $5.7509 \mathrm{E}-05$ & 5.2961 & 5.78614 & 7.87 & \\
$\mathrm{E}$ & $1.3948 \mathrm{E}-04$ & 2 & $6.9740 \mathrm{E}-05$ & 6.4225 & 5.78614 & 9.54 & 3 \\
$\mathrm{~F}$ & $5.2626 \mathrm{E}-04$ & 2 & $2.6313 \mathrm{E}-04$ & 24.2322 & 5.78614 & $\mathbf{3 6 . 0 1}$ & 1 \\
\hline Sai số & $5.4294 \mathrm{E}-04$ & 5 & $1.0859 \mathrm{E}-05$ & & & & \\
Tổng cộng & 0.00146 & 17 & & & & & \\
\hline
\end{tabular}

\subsection{Mô phỏng kiểm tra xác thực}

Từ phân tích tỷ lệ $\mathrm{S} / \mathrm{N}$, biến dạng tối thiểu có thể thu được dưới sự kết hợp của $\mathrm{A} 1-\mathrm{B} 3-\mathrm{C} 1-\mathrm{D} 3-\mathrm{E} 1-\mathrm{F} 3$ như được chỉ ra trong Hình 5 . Từ hình dạng của lưỡi cắt, sự kết hợp này mở rộng đầu dao băm, và do đó giảm thiểu sự biến dạng. Kết quả này được kiểm tra xác thực bằng phần mềm Minitab ${ }^{\circledR}$. Kết quả xác thực (Hình 7) cho thấy sự nhất quán với kết quả tính thủ công bằng Excel®. Điều này cho thấy tính chính xác của quá trình vận dụng công thức. Thực tiễn cho thấy, các kỹ sư trong các phân xưởng sản xuất chỉ vận dụng phần mềm, chẳng hạn như Minitab ${ }^{\circledR}$, để tối thiểu hóa thời gian quy hoạch thực nghiệm và phân tích kết quả.

Một mô phỏng bổ sung đã được thực hiện bằng cách sử dụng các điều kiện này như một thử nghiệm xác nhận. Kết quả cho thấy độ biến dạng tối đa là $0,16209 \mu \mathrm{m}$, Hình 8 . Giá trị này thấp hơn giá trị biến dạng của tất cả các trường hợp được liệt kê trong mảng trực giao trong Bảng 3 


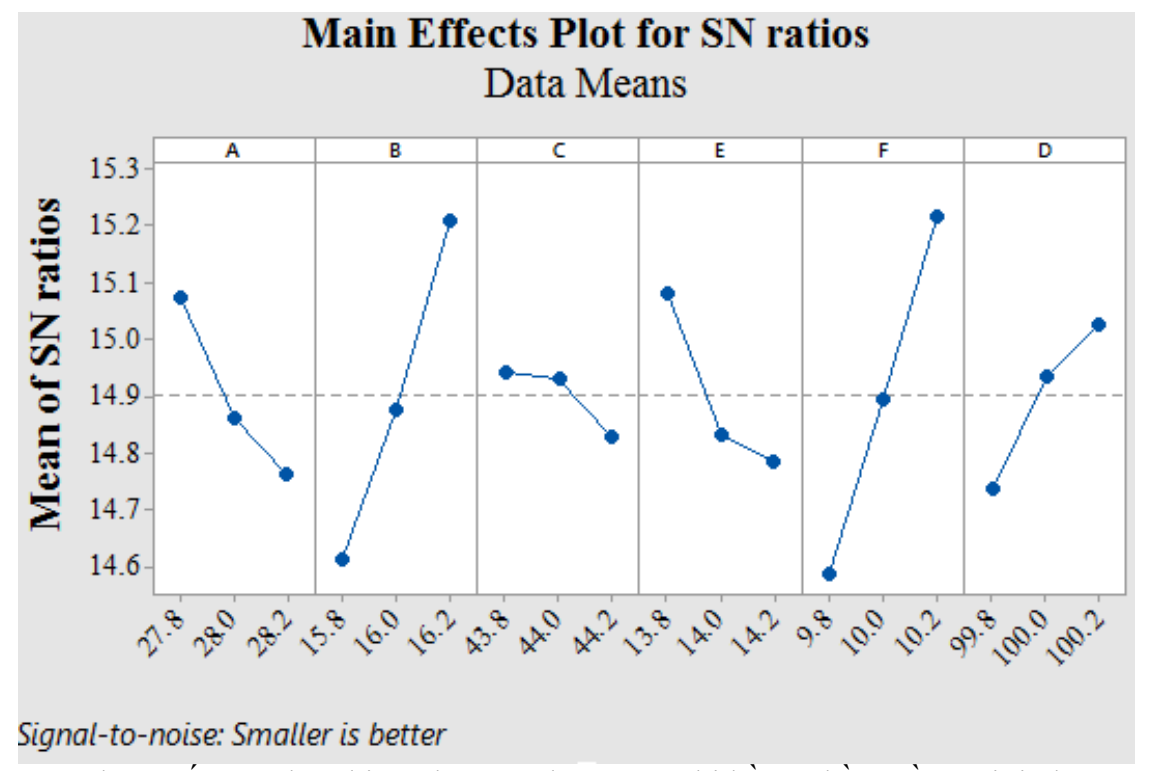

Hình 7. Kết quả thực hiện phương pháp Taguchi bằng phần mềm Minitab®.
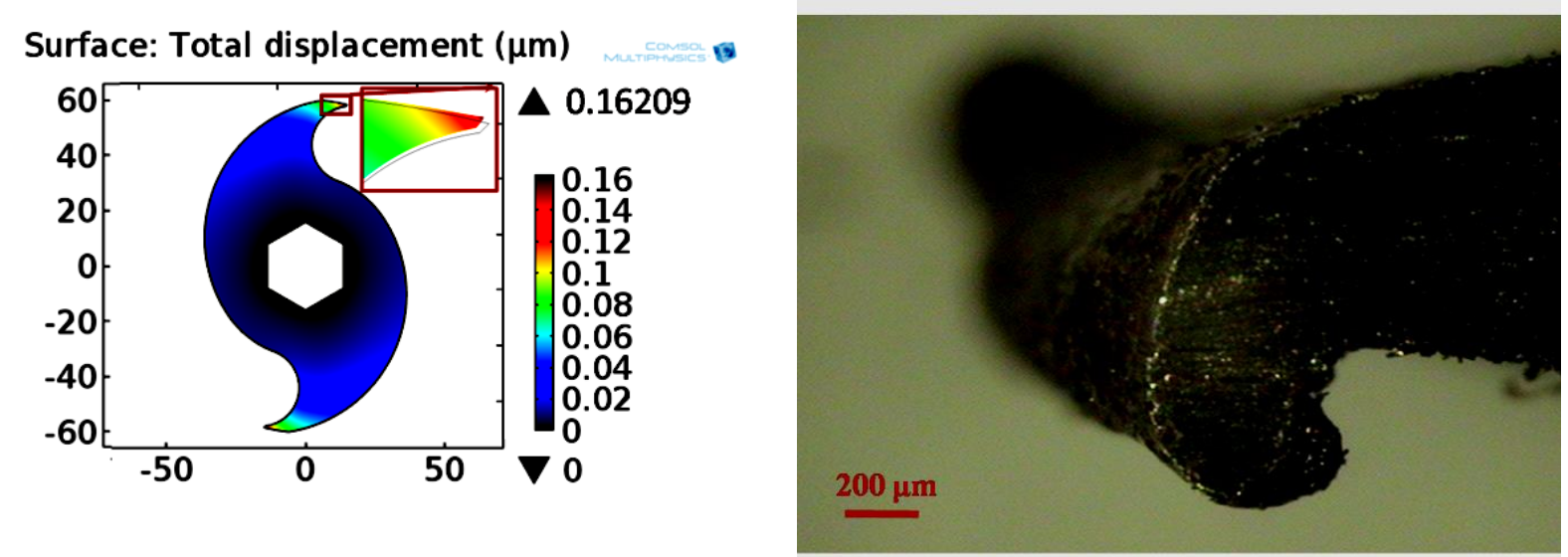

Hình 8. Phân bố của biến dạng của dao có các yếu tố hình Hình 9 . Hình ảnh lưỡi cắt được sử dụng trước đây thông học đã được tối ưu hóa qua Icamscope $®$

\subsection{Thực nghiệm kiểm tra xác thực}

Để xác thực các yếu tố hình học tối ưu thu được bằng phương pháp Taguchi, một thí nghiệm xác nhận đã được thực hiện. Trong thí nghiệm, hai loại lưỡi cắt, được đề xuất từ phương pháp Taguchi và dao cũ, đã được sử dụng đồng thời. Điều đáng chú ý là các lưỡi dao băm cũ, được sử dụng trước đây trong xưởng, đã bị biến dạng dễ dàng như trong Hình 9 sau khoảng năm giờ làm việc liên tục. Hai loại lưỡi dao băm mới sau đó cũng được đo bằng kính hiển vi điện tử sau khoảng năm giờ làm việc liên tục. Cần lưu ý rằng biến dạng của mũi dao là biến dạng không đẳng hướng. Các đầu mũi dao trong các Hình 9,10 và 11 đã được mài phẳng trước khi đo. Trước khi cải tiến, mũi dao trong Hình 9 thể hiện sự biến dạng bất đẳng hướng rất rõ rệt, kết quả biến dạng này không thể đo và phân tích định lượng. Sau khi cải tiến, mũi dao cứng chắc hơn, các biến dạng chỉ còn rất nhỏ (sau 5 giờ hoạt động liên tục). Do đó, sự thay đồi bán kính đầu dao được lựa chọn là thông số đánh giá sự biến dạng của dao.

Các Hình $10 \mathrm{a}$ và $\mathrm{b}$ lần lượt hiển thị kích thước của đầu băm của lưỡi cắt từ dao số \#3 trong Bảng 3 và dao có biên dạng theo đề xuất từ phương pháp Taguchi trước khi chạy thử nghiệm. Kích thước của chúng gần như giống nhau, $63 \mu \mathrm{m}$ so với $62 \mu \mathrm{m}$. Còn các Hình $11 \mathrm{a}$ và b hiển thị kích thước của chúng sau khoảng năm giờ làm việc liên tục. Bán kính của đầu băm của lưỡi dao băm số \#3 tăng lên $88 \mu \mathrm{m}$ trong khi của lưỡi dao từ Taguchi là $71 \mu \mathrm{m}$. Từ thử nghiệm xác nhận này, ta có thể kết luận được rằng phương pháp Taguchi có thể tạo ra một sự kết hợp các thông số kích thước hình học tốt hơn để có thể giảm thiểu độ biến dạng của dao băm. 


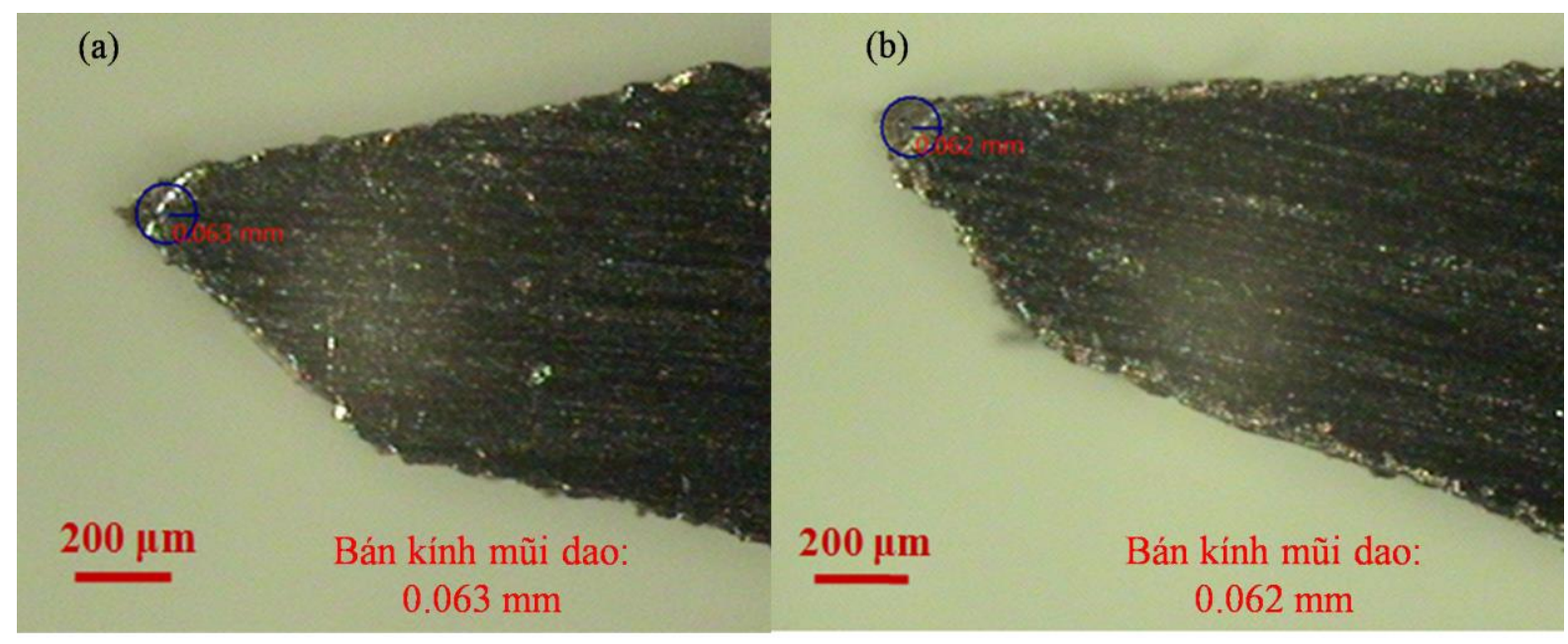

Hình 10. Ảnh chụp lưỡi dao băm trước khi thử nghiệm qua Icamscope ${ }^{\circledR}$ (a) trường hợp \#3 (b) sau khi dùng Taguchi

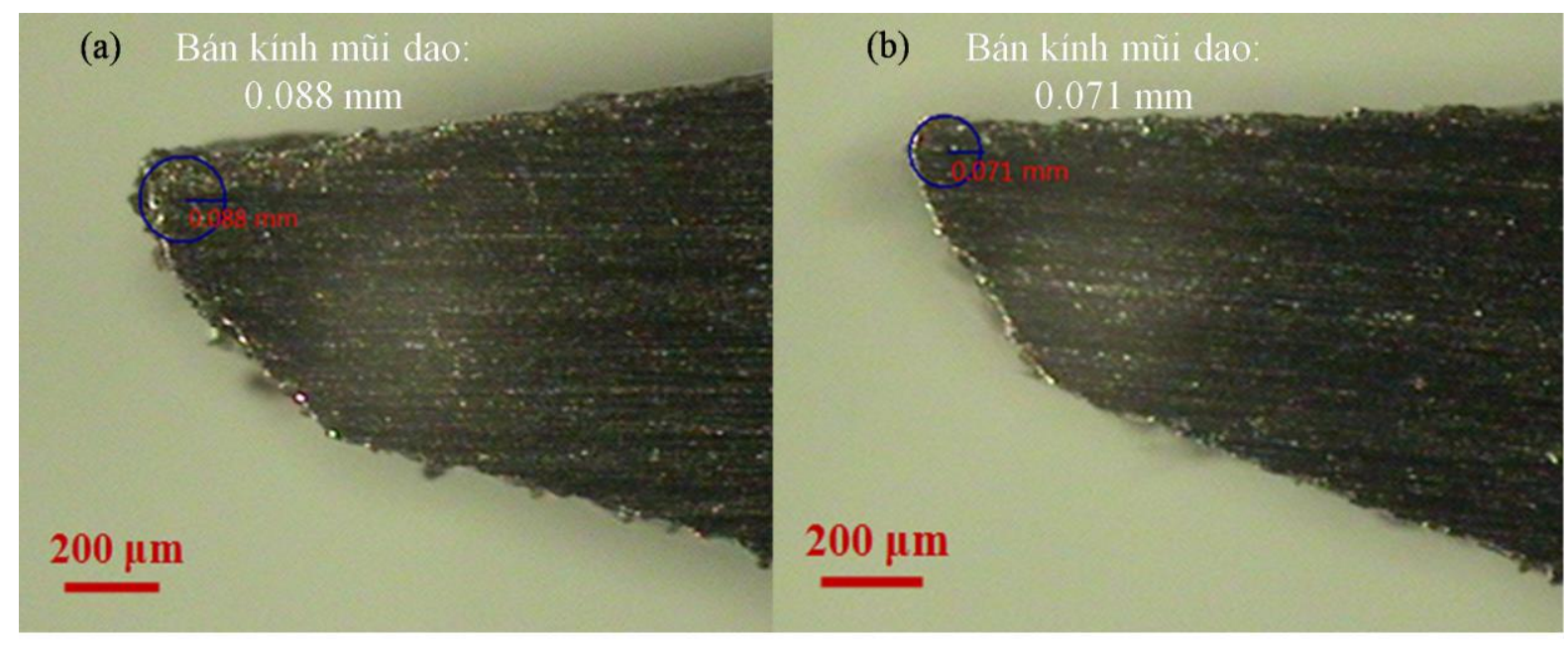

Hình 11. Ảnh chụp lưỡi dao băm sau khi thử nghiệm qua Icamscope ${ }^{\circledR}$ (a) trường hợp \#3 (b) sau khi dùng Taguchi

Do đó, các kết quả đã xác nhận cả bằng mô phỏng lẫn thực nghiệm, rằng phương pháp Taguchi là đủ để khắc phục nhược điểm về hình học của lưỡi dao băm, cho kết quả tốt hơn trong phạm vi cho phép mà không cần sử dụng các thuật toán hoặc phần mềm phức tạp khác.

\section{KẾT LUẬn VÀ ĐỀ NGH!}

Trong nghiên cứu hiện tại, phương pháp Taguchi đã được sử dụng để nghiên cứu ảnh hưởng của các yếu tố hình học đến sự biến dạng của lưỡi cắt của máy băm chai nhựa và cũng để xác định sự kết hợp tối ưu cho sự biến dạng tối thiểu. Kỹ thuật tối ưu hóa đơn giản được sử dụng trong nghiên cứu này về cơ bản bao gồm kỹ thuật mảng trực giao của phương pháp Taguchi được sử dụng kết hợp với phân tích phương sai ANOVA.

Trong các mô phỏng cho lưỡi dao băm với tải trọng băm cần thiết, sáu yếu tố hình học đã được xem xét. Từ phân tích tỷ lệ $\mathrm{S} / \mathrm{N}$ và $\mathrm{ANOVA}$ dựa trên phương pháp Taguchi với mảng trực giao $\mathrm{L}_{18}\left(3^{6}\right)$, tọa độ $\mathrm{y}$ $\Phi 100 \mathrm{~mm}$ và tọa độ $\mathrm{x} \Phi 28 \mathrm{~mm}$ là các yếu tố quan trọng nhất để giảm thiểu độ biến dạng của đầu lưỡi dao băm. Sau đó, kết quả tối ưu hóa đã được kiểm chứng thông qua cả mô phỏng lẫn thực nghiệm.

Sự đơn giản và khả năng của phương pháp tối ưu hóa được sử dụng trong nghiên cứu này chứng minh tính tiện ích của nó trong các công nghệ sản xuất khác nhau của các nhà máy quy mô vừa và nhỏ, nơi không phải lúc nào cũng có các công cụ tối ưu hóa thương mại. Sự hữu ích của phương pháp hiện tại cũng được hy vọng sẽ góp phần kéo nghiên cứu học thuật đến gần thực tiễn sản xuất công nghiệp hơn.

Tuy nhiể, do tính chất rời rạc của phương pháp Taguchi, kết quả tối ưu hóa từ phương pháp này bị ràng buộc, chỉ là một trong ba mức đã khảo sát. Do đó, để tìm một kết quả tối ưu hơn nữa, phương pháp bề mặt đáp ứng RSM sẽ được áp dụng để phát triển nghiên cứu này. 


\section{LÒ̀I CẢM ƠN}

Công trình nghiên cứu này được tài trợ bởi quỹ nghiên cứu khoa khọc của trường đại học Công Nghiệp TP.HCM, theo dự án nghiên cứu khoa học cấp trường số 21/1CK01. Quá trình đo dùng kính hiển vi điện tử ICamScope ${ }^{\circledR}$ được thực hiện tại Phòng thí nghiệm Công nghệ thiết kế và gia công tiên tiến, Đại học Bách Khoa thành phố Hồ Chí Minh.

\section{TÀI LIẸU THAM KHẢO}

[1] S. Alavi, S. Thomas, K. P. Sandeep, N. Kalarikkal, J. Varghese, and S. Yaragalla, Polymers for Packaging Applications: Apple Academic Press, 2014.

[2] A. Ayo, O. J. Olukunle, and D. J. Adelabu, International Journal of Waste Resources, vol. 7, no. 1, pp. 1-4, 2017.

[3] P. K. Farayibi, Nigerian Journal of Technology, vol. 36, no. 2, p. 411-420, 2017.

[4] S. Reddy and T. Raju, Design and development of mini plastic shredder machine, in IOP Conference Series: Materials Science and Engineering, 2018, vol. 455, p. 012119.

[5] D. Atadious and O. Joel, International Journal of Scientific and Engineering Research, vol. 9, no. 5, pp. 1379$1385,2018$.

[6] Y. M. Sonkhaskar, A. Sahu, A. Choubey, A. Singh, and R. Singhal, International Journal of Engineering Research \& Technology, vol. 3, no. 10, pp. 297-300, 2014.

[7] M. I. Faiyyaj, M. R. Pradip, B. J. Dhanaji, D. P. Chandrashekhar, and J. S. Shivaji, International Journal of Engineering Technology Science and Research, vol. 4, no. 10, pp. 733-737, 2017.

[8] S.B.Satish, J. S. Sandeep, B.Sreehari, and Y. M. Sonkhaskar, International Journal for Scientific Research \& Development, vol. 4, no. 7, pp. 891-893, 2016.

[9] N. D. Jadhav, A. Patil, H. Lokhande, and D. Turambe, International Journal of Waste Resources, vol. 08, no. 2, pp. 1-4, 2018.

[10] T. A. Olukunle, International Journal of Industrial and Manufacturing Engineering, vol. 10, no. 11, pp. 18381841, 2016.

[11] A. Tegegne, A. Tsegaye, E. Ambaye, and R. Mebrhatu, International Journal of Research and Scientific Innovation, vol. 6, no. 1, pp. 49-55, 2019.

[12] J. M. A. Jaff, D. A. Abdulrahman, Z. O. Ali, K. O. Ali, and M. H. Hassan, International Journal of Scientific \& Engineering Research, vol. 7, no. 5, pp. 1471-1486, 2016.

[13] S. Yadav, S. Thite, N. Mandhare, A. Pachupate, and A. Manedeshmukh, Journal of Applied Science and Computations, vol. 4, no. 10, pp. 21-25, 2019.

[14] S. Ravi, Utilization of Upgraded Shredder Blade and Recycling the Waste Plastic and Rubber Tyre, in International Conference on Industrial Engineering and Operations Management, Paris, France, 2018, pp. 32083216.

[15] M. F. Nasr and K. A. Yehia, Journal of International Society for Science and Engineering, vol. 1, no. 1, pp. 9$12,2019$.

[16] C. P. Yepes, P. R. M. Angel, and P. G. Jose, Contemporary Engineering Sciences, vol. 11, no. 83, pp. 41134120, 2018.

[17] A. Ikpe and O. Ikechukwu, International Journal of Engineering Technologies IJET, vol. 3, no.3, pp. 157-168, 2017. 
[18] T. K. Nguyen, C. J. Hwang, and B.-K. Lee, International Journal of Precision Engineering and Manufacturing, vol. 18, pp. 187-195, 2017.

[19] G. Taguchi, S. Chowdhury, and Y. Wu, Introduction to the Signal-to-Noise Ratio in Taguchi's Quality Engineering Handbook, ed. 2004, pp. 221-238, 2004.

[20] N. K. Triều, N. C. Thông, N. V. Trung, T. A. Tuấn, N. Q. Vinh, and T. T. Vũ, Tạp chí Khoa học và công nghệ Trừ̀ng đại học Công nghiệp Thành phố Hồ Chí Minh, vol. 40, pp. 70-82, 2019.

[21] G. Taguchi, S. Chowdhury, and Y. Wu, Fundamentals of Data Analysis in Taguchi's Quality Engineering Handbook, pp. 506-514, 2004.

Ngày nhận bài: 23/03/2020

Ngày chấp nhận đăng: 11/08/2021 